\title{
REGULAR OPERATOR EQUATIONS: CONDITIONS FOR REGULARITY ${ }^{1}$
}

\author{
GEORGE KARAKOSTAS ${ }^{2}$
}

\begin{abstract}
Regular operator equations are causal equations admitting unique solutions and have the property that all of their limiting equations along solutions admit unique solutions. Sufficient conditions which guarantee that an operator equation $x=T x$ is regular are given in case $T$ is a linear or a nonlinear operator.
\end{abstract}

Introduction. Regular operator equations are causal equations with unique solutions and such that all of their limiting equations along solutions admit unique solutions. In this paper we give sufficient conditions which guarantee that an operator equation

$$
x=T x
$$

is regular. Conditions for uniqueness of the solutions of causal operator equations of the form (*) have been suggested by Neustadt [7] but such conditions are not enough for regularity. Furthermore there is the problem of uniqueness of the solutions of the limiting equations of $(*)$ (whenever such equations exist) as they were defined in [5]. (We shall refer to the basic definitions in $§ 1$.) Sell first discussed this problem in case (*) is generated from the nonautonomous ordinary differential equation $\dot{x}=f(x, t)$, see [8]. Namely, Sell investigated when such a function $f$ is "regular" (in the terminology of [8]). The hypothesis of regularity was also used in [1] (but under a weaker sense of the word). In [6], where Volterra integral equations are studied, a uniqueness assumption inherited by the limiting equations along solutions is essential.

Our idea applied here is rather simple and is inspired from an analogous one of Sell [8]: a Lipschitz condition imposed on the original operator is inherited by the translations of the operator along any function, as they were defined in [4]. Thus such a Lipschitz condition also governs any limiting operator. The uniqueness will follow from the basic Lemma 2.6. In the case of linear causal operators a more intrinsic sufficient condition guarantees regularity (Theorem 2.1).

1. Some basic preliminaries. We start with some basic definitions and notation, some of them borrowed from $[4,5]$, and with some auxiliary results.

Received by the editors February 2, 1981 and, in revised form, July 24, 1981.

1980 Mathematics Subject Classification. Primary 47H15, 47B38.

'This paper is based on the author's Ph. D. Thesis written under the supervision of Professor Zvi Artstein of the Weizmann Institute of Science, Rehovot, Israel, 1979.

${ }^{2}$ This research was supported by the Deutscher Akademischer Austauschdienst. 
We shall denote by $C_{n}$ the space of all continuous functions $\varphi:[0, \infty) \rightarrow \mathbf{R}^{n}$ endowed with the compact-open topology. For any $\xi \in \mathbf{R}^{n}$ the symbol $C_{n}(\xi)$ denotes the set $\left\{\varphi \in C_{n}: \varphi(0)=\xi\right\}$. For any $\tau>0$ let $C_{n}^{\tau}$ denote the set of all continuous $\psi$ : $[0, \tau] \rightarrow \mathbf{R}^{n}$ considered endowed with the sup-norm $\|\cdot\|_{\tau}$.

Following [7] we say that an operator $T: C_{n} \rightarrow C_{n}$ is causal if for all $t \geqslant 0$ and $x, y$ in $C_{n}$ we have $(T x)(t)=(T y)(t)$, whenever $x(s)=y(s), s \leqslant t$. Let $T$ be a causal operator and let $\tau>0$. Then by

$$
\left(T^{\tau} \varphi\right)(t)=(T \bar{\varphi})(t), \quad t \in[0, \tau], \varphi \in C_{n}^{\tau},
$$

where $\bar{\varphi}$ is any continuation of $\varphi$ on $(\tau, \infty)$, a new operator $T^{\tau}: C_{n}^{\tau} \rightarrow C_{n}^{\tau}$ is well defined. Finally we recall that a set $B \subseteq C_{n}$ is bounded with respect to the above (metrizable) topology of $C_{n}$ if there exists a $p \in C_{1}$ such that $|\varphi(t)| \leqslant p(t), t \geqslant 0$, for all $\varphi \in B$.

The following lemma is obvious.

LEMMA 1.1. A causal operator $T: C_{n} \rightarrow C_{n}$ is compact if and only if for all $\tau>0$ the operator $T^{\tau}$ is compact.

Let now $\mathbf{T}(v)$ be the set of all continuous causal operators on $C_{n}(v)$ to $C_{n}$, for every $v \in \mathbf{R}^{n}$, and let $\mathbf{T}$ denote the set $\cup_{v \in \mathbf{R}^{n}} \mathbf{T}(v)$. Thus for each $T \in \mathbf{T}$ there exists a unique $v=v(T) \in \mathbf{R}^{n}$ such that $T \in \mathbf{T}(v)$. Following [4] we say that a sequence $\left\{T^{m}\right\} \subset \mathbf{T}$ converges to an operator $T \in \mathbf{T}$ if $v\left(T^{m}\right) \rightarrow v(T)$ and for any sequence $\left\{\varphi_{m}\right\} \subset C_{n}$ such that $\varphi_{m} \in C\left(v\left(T^{m}\right)\right), m=1,2, \ldots$, and $\varphi_{m} \rightarrow \varphi$, for some $\varphi \in C_{n}$, we have $\varphi \in C(v(T))$ and $T^{m} \varphi_{m} \rightarrow T \varphi$.

According to [5] the limiting equations along solutions of $(*)$ are defined via the translations of (*) along solutions as follows: let $T \in \mathrm{T}$ be such that there exists an $x \in C(v(T))$ satisfying $x=T x$. Such operators are called "admissible" operators. Let $\tau \geqslant 0$ and let $x$ be any function in $C$. Denote by $x_{\tau}$ the function $x_{\tau}(s)=$ $x(\tau+s), s \geqslant 0$. For any $\varphi \in C(\mathrm{x}(\tau))$ define $\mu_{\tau, \mathrm{x}} \varphi$ in $C$ by $\mu_{\tau, x} \varphi(t)=x(t), t \leqslant \tau$, and $\mu_{\tau, x} \varphi(t)=\varphi(t-\tau), t>\tau$. The "translation $T_{\tau, x}$ of $T$ along $x$ by $\tau^{\prime \prime}$ is defined by $T_{\tau, x} \varphi=\left(T \mu_{\tau, x} \varphi\right)_{\tau}$. Now the limiting equations of $(*)$ along the solution $x$ are the equations of the form $y=S y$, where $S=\lim T_{t_{m}, x}$, for a certain $t_{m} \rightarrow \infty$ such that $\left\{x_{t_{m}}\right\}$ converges in $C_{n}$. The function to which the sequence $\left\{x_{t_{m}}\right\}$ converges is clearly a solution of $y=S y$.

A causal operator $T$ is called regular if (*) admits a unique solution $x$ and all limiting equations of $(*)$ along $x$ admit unique solutions.

Notice that if $x=T x$ admits a unique solution $x$ then for any $\tau \geqslant 0$ the equation $u=T_{\tau, x} u$ also admits a unique solution which must be the function $x_{\tau}$ (see [4, Example 3.7]). Moreover the uniqueness property might not be inherited by the limiting equations along solutions. For an example see Remark 2.3. Another example is given in [8, Example D] where the translations of the generated integral operator can be taken along $x(t)=0, t \geqslant 0$.

2. The main results. We shall discuss the case of linear operators in $\mathbf{T}(0)$ first and then the general case. We note that the norm of a bounded linear operator $S$ : $X \rightarrow X$, where $X$ is a normed linear space, is defined by $|S|=\sup _{\|u\|=1}\|S u\|$. The identity operator on $C_{n}^{\tau}$ (and on $C_{n}^{\tau}(\xi)$ ) will be denoted by $I^{\tau}$. 
Theorem 2.1. Let $L$ be a linear compact operator in $\mathbf{T}(0)$. If there exists an $M<\infty$ such that

$$
\left|\left(I^{\tau}-L^{\tau}\right)^{-1}\right| \leqslant M, \quad \tau>0,
$$

then the operator $L$ is regular.

The proof of the theorem relies on the following result due to Neustadt.

TheOREM 2.2 (NeUSTADT [7]). Consider $a \tau>0$ and an affine operator $A: C_{n}^{\tau} \rightarrow C_{n}^{\tau}$ satisfying the following conditions:

(i) $x_{1}(0)=\left(A x_{1}\right)(0)$, for a certain $x_{1} \in C_{n}^{\tau}$,

(ii) $A$ is causal,

(iii) $(A \varphi)(0)=(A \psi)(0)$, for all $\varphi, \psi$ in $C_{n}^{\tau}\left(x_{1}(0)\right)$, and

(iv) $A$ is continuous and compact on $C_{n}^{\tau}\left(x_{1}(0)\right)$.

Then the equation $x=A x$ admits a unique solution in $C_{n}^{\tau}\left(x_{1}(0)\right)$.

Proof of Theorem 2.1. We extend $L$ on $C_{n}$ linearly by setting $L_{1} \varphi=L(\varphi-\varphi(0))$, $\varphi \in C_{n}$. Then $L_{1} \varphi=L \varphi, \varphi \in C_{n}(0)$, and Theorem 2.2 is applicable with $x_{1}$ the zero function. Note that the causality of $L$ implies $(L \varphi)(0)=0$, for all $\varphi \in C_{n}(0)$. Thus the equation $u=L^{\tau} u$ admits the unique solution $u=0$, for all $\tau>0$. (This means that the mapping $I^{\tau}-L^{\tau}: C_{n}^{\tau}(0) \rightarrow C_{n}^{\tau}(0)$ is a one-to-one linear mapping and, by [2, Theorem 2, p. 57], its inverse $\left(I^{\tau}-L^{\tau}\right)^{-1}$ is a continuous linear operator on $C_{n}^{\tau}(0)$ to $C_{n}^{\tau}(0)$. So (2.1) makes sense.)

We claim that the operator equation $u=L u$ admits a unique solution $x$, thus $x \equiv 0$. Indeed, let $x \neq 0$ be a solution and let $\tau>0$. Define $x^{\tau}(t)=x(t), t \leqslant \tau$, and then observe that $x^{\tau}=L^{\tau} x^{\tau}$. By the above arguments we must have $x^{\tau}(t)=0, t \leqslant \tau$. Since $\tau$ is arbitrary, it follows that $x(t)=0, t \geqslant 0$.

Let now $u=S u$ be a limiting equation of $x=L x$ along $x=0$. Then $0=S 0$ and there is a sequence $\left\{t_{k}\right\} \subset \mathbf{R}^{+}$such that $t_{k} \rightarrow \infty$ and $L_{t_{k}, 0} \rightarrow S$. We assume that the equation $u=S u$ admits a nonzero solution $x \in C_{n}(0)$. Hence there is an $r>0$ such that $x(r) \neq 0$. By the linearity of $S$ we can assume that $\left\|x^{r}\right\|_{r}=1$, where, recall that $x^{r}$ is the restriction of $x$ on $[0, r]$. Since $\left\|x^{r}-S^{r} x^{r}\right\|_{r}=0$, there exists a term $t$ of the sequence $\left\{t_{k}\right\}$ such that

$$
\left\|x^{r}-\left(L_{t, 0} x\right)^{r}\right\|_{r} \leqslant 1 / 2 M,
$$

where $M$ is a number satisfying (2.1). We notice that $x \in C_{n}(v(S))=C_{n}(0)=$ $C_{n}\left(v\left(L_{s, 0}\right)\right)$, for all $s \geqslant 0$. Set $y=\mu_{t, 0} x$ and $\tau=t+r$. Then clearly $\left\|y^{\tau}\right\|_{\tau}=\left\|x^{r}\right\|_{r}$ $=1$ and

$$
\begin{aligned}
\left\|x^{r}-\left(L_{t, 0} x\right)^{r}\right\|_{r} & =\sup _{s \in[0, r]}\left|x(s)-\left(L_{t, 0} x\right)(s)\right|=\sup _{s \in[0, \tau]}|y(s)-(L y)(s)| \\
& =\left\|y^{\tau}-L^{\tau} y^{\tau}\right\|_{\tau} \geqslant\left|\left(I^{\tau}-L^{\tau}\right)^{-1}\right|^{-1}\left\|y^{\tau}\right\|_{\tau}=\left|\left(I^{\tau}-L^{\tau}\right)^{-1}\right|^{-1} .
\end{aligned}
$$

Thus by (2.1) we get $\left\|x^{r}-\left(L_{t, 0} x\right)^{r}\right\|_{r} \geqslant 1 / M$, which contradicts (2.2). Therefore $x \equiv 0$ and the proof of the theorem is complete.

REMARK 2.3. If compactness is preserved in taking limits of a sequence of translations of $L$ along the function $x=0$, then by Theorem $2.2, L$ would be a 
regular operator. From this fact and since the identity operator is not regular, it follows that the linear operator

$$
(L \varphi)(t)=t \int_{0}^{t} e^{-t(t-s)} \varphi(s) d s, \quad \varphi \in C_{n}(0)
$$

must not satisfy the condition (2.1). Notice that $L_{\tau, 0} \rightarrow I$, the identity on $C_{n}$ (see [4]). We shall check it by following a different way. Consider the sequence of functions $\varphi_{m}(t), t \in[0, m], m=1,2, \ldots$, defined by

$$
\varphi_{m}(t)= \begin{cases}0, & t \leqslant m-1, \\ t-m+1, & t \in(m-1, m], \quad m=1,2, \ldots\end{cases}
$$

We then observe that $\left\|\varphi_{m}\right\|_{m}=1$ and $\left\|\left(I^{m}-L^{m}\right) \varphi_{m}\right\|_{m} \leqslant 1 /(m-1)$. Therefore there does not exist any $M \in(0, \infty)$ such that $(2.1)$ is satisfied.

An operator $R: \mathrm{C}_{1} \rightarrow C_{1}$ is called increasing if $(R q)(t) \leqslant(R p)(t), t \geqslant 0$, whenever $q(t) \leqslant p(t), t \geqslant 0$. It is obvious that if $R$ is linear, then $R$ is increasing if and only if it is positive, i.e., $(R p)(t) \geqslant 0, t \geqslant 0$, whenever $p(t) \geqslant 0, t \geqslant 0$.

THEOREM 2.4. Let $T$ be an admissible operator and let $x$ be a solution of the operator equation $u=T u$. Assume that for each $K>0$ there exists a positive continuous compact linear causal operator $L: C_{1} \rightarrow C_{1}(0)$ such that

$$
|(T x)(t)-(T y)(t)| \leqslant L(|x-y|)(t), \quad t \geqslant 0,
$$

for all $y$ in $C_{n}(v(T))$ with $|x(s)-y(s)| \leqslant K$, for all $s \geqslant 0$; where $|x-y|$ is the function $|x(t)-y(t)|, t \geqslant 0$. Assume also that whenever a sequence $\left\{\left(x_{t_{n}}, T_{t_{n}, x}\right)\right\}$ converges in $C_{n} \times \mathbf{T}$ then $\left\{L_{t_{n}, 0}\right\}$ is precompact. If $L$ satisfies the condition (2.1) for a certain $M<\infty$, then the operator $T$ is regular.

REMARK 2.5. The local Lipschitz condition (2.3) is inspired by an analogous condition given in [8] concerning nonautonomous ordinary differential equations.

To prove the theorem, we need the following lemma.

LEMMA 2.6. Suppose that an operator $L$ is as in the preceding theorem. Then for any operator $N$ in the (sequential) closure of the set $\left\{L_{t, 0}: t \geqslant 0\right\}$ the relation

$$
0=q(0) \leqslant q(t) \leqslant(N q)(t), \quad t \geqslant 0,
$$

implies that $q(t)=0, t \geqslant 0$.

Proof. We first observe that, for any $t \geqslant 0$, the operator $L_{t, 0}$ is defined on $C_{1}(0)$, it is causal, and for any $\tau>0$ the operator $L_{t, 0}^{\tau}: C_{1}^{\tau}(0) \rightarrow C_{1}^{\tau}(0)$ is linear, positive, bounded, compact (by Lemma 1.1) and satisfies the condition

$$
\left|\left(I^{\tau}-L_{t, 0}^{\tau}\right)^{-1}\right| \leqslant M
$$

where $M$ is a constant satisfying (2.1). Let $r>0$ be fixed and let $R=L_{r, 0}$. We claim that the operator $R$ has an extension $\hat{R}$ on $C_{1}$ to $C_{1}(0)$ such that for all $\tau>0$ the operator $\hat{R}^{\tau}: C_{1}^{\tau} \rightarrow C_{1}(0)$ is causal, linear, positive, bounded, compact and satisfies (2.5). 
Indeed, let $\tau>0$ and let $\varphi \in C_{1}^{\tau}$. For any integer $k$ with $k \tau>1$ define a function $\varphi_{k} \in C_{1}^{\tau}(0)$ by

$$
\varphi_{k}(t)=\left\{\begin{array}{ll}
\varphi(t), & t>1 / k, \\
k t \varphi(1 / k), & t \leqslant 1 / k,
\end{array} \quad t \leqslant \tau .\right.
$$

Set $z_{k}(t)=\mu_{r, 0} \varphi_{k}(t), t \in[0, \tau+r]$. Then $\left\{z_{k}\right\}$ is bounded in $C_{1}^{\tau+r}$ and by the compactness of $L^{\tau+r}$, the sequence $\left\{L^{\tau+r} z_{k}\right\}$ has a limit point $\psi$ in $C_{1}^{\tau+r}(0)$. If $\varphi(0)=0$, then, for all $k$, we can verify that

$$
\left\|L^{\tau+r} z_{k}-L^{\tau+r} \mu_{r, 0} \varphi\right\| \leqslant\left|L^{\tau+r}\right|\left(\left|\varphi\left(\frac{1}{k}\right)\right|+\sup _{0 \leqslant s \leqslant 1 / k}\left|\varphi\left(\frac{1}{k}\right)-\varphi(s)\right|\right)
$$

which tends to zero, as $k \rightarrow \infty$. Thus $\psi=L^{\tau+r} \mu_{r, 0} \varphi$. Suppose that $\varphi(0) \neq 0$. We shall show that $\psi$ is the unique limit point of $\left\{L^{\tau+r} z_{k}\right\}$, thus by the monotonicity of $L^{t+r}$, $\psi$ is its limit. To do this, assume that $\left\{z_{n_{k}}\right\},\left\{z_{m_{k}}\right\}$ are two subsequences of $\left\{z_{k}\right\}$ with $L^{\tau+r} z_{n_{k}} \rightarrow \psi$ and $L^{\tau+r_{z}} z_{m_{k}} \rightarrow \psi_{1}$, for a certain $\psi_{1} \in C_{1}^{\tau+r}(0)$. We can assume that $n_{k+1}>m_{k}>n_{k}$, for all $k$. Thus for all large $k$ and all $t \in[0, \tau+r]$ we have $z_{n_{k+1}}(t) \geqslant z_{m_{k}}(t) \geqslant z_{n_{k}}(t)$, if $\varphi(0)>0$ and $z_{n_{k+1}}(t) \leqslant z_{m_{k}}(t) \leqslant z_{n_{k}}(t)$, if $\varphi(0)<0$. Since $L^{\tau+r}$ is increasing we can easily get that $\psi=\psi_{1}$. Thus $\psi$ is unique, namely, $\psi=\lim L^{\tau+r} z_{k}$. So we can define an operator $\hat{R}^{\tau}: C_{1}^{\tau} \rightarrow C_{1}^{\tau}$ by setting $\hat{R}^{\tau} \varphi=\psi_{r}=$ $\lim L_{r, 0}^{\tau+r} \varphi_{k}$ and $\varphi_{k}$ is defined via $\varphi$ as above. By (2.6) it follows that $\hat{R}^{\tau} \varphi=R^{\tau} \varphi$, for all $\varphi \in C_{1}^{\tau}(0)$, thus $\hat{R}^{\tau}$ is an extension of $R^{\tau}$ on $C_{1}^{\tau}$. The remaining properties, which we earlier promised $\hat{R}^{\tau}$ would have, can easily be checked by using the corresponding properties of the operator $R^{\tau}$. Now by the causality of $\hat{R}^{\tau}$, for all $\tau>0$, the type $(\hat{R} \varphi)(t)=\left(\hat{R}^{\tau} \varphi^{\tau}\right)(t), t \geqslant 0, t \leqslant \tau$, with $\varphi^{\tau}(t)=\varphi(t), t \leqslant \tau$, defines a causal operator $\hat{R}: C_{1} \rightarrow C_{1}(0)$ with the desired properties and our claim is proved.

Let $N$ be an operator which satisfies the requirements of the lemma and let $q$ be a function in $C_{1}(0)$ satisfying (2.4). By Theorem 2.1 the operator $L$ is regular and therefore the equation

$$
\varepsilon=r+N \varepsilon,
$$

where $r=N q-q$, admits a unique solution, the function $\varepsilon=-q$. Let $\left\{t_{k}\right\} \subset \mathbf{R}^{+}$be a sequence such that $L_{t_{k}, 0} \rightarrow N$, as $k \rightarrow \infty$. Let $\hat{L}_{t_{k}, 0}$ be the extension of $L_{t_{k}, 0}$ on $C_{1}$ obtained by the preceding manner. Fix a $\tau>0$ and set $r^{\tau}(t)=r(t)=(N q)(t)-$ $q(t), t \in[0, \tau]$. Then Theorem 2.2 applies to the equation

$$
u=r^{\tau}+\frac{1}{k}+\hat{L}_{t_{k}, 0}^{\tau} u, \quad k=1,2, \ldots,
$$

(with $x_{1}=1 / k$ ) and ensures that it admits a unique solution $p_{k}$ in $C_{1}^{\tau}(1 / k)$ given by

$$
p_{k}=\left(I^{\tau}-\hat{L}_{t_{k}, 0}\right)^{-1}\left(r^{\tau}+\frac{1}{k}\right), \quad k=1,2, \ldots
$$

Since $r(t)+1 / k>0$, for all $t \in[0, \tau]$, by Theorem 4.3 in [7], we conclude that $p_{k}(t) \geqslant 0$, for all $t \in[0, \tau]$ and $k=1,2, \ldots$ On the other hand if $\varepsilon^{\tau}$ is the restriction on $[0, \tau]$ of the solution $\varepsilon$ of $(2.7)$, we claim that

$$
p_{k} \rightarrow \varepsilon^{\tau}, \quad \text { as } K \rightarrow \infty \text {. }
$$


Indeed, by (2.5), (2.7) and (2.8) we get

$$
\begin{aligned}
\left\|p_{k}-\varepsilon^{\tau}\right\|_{\tau} & =\left\|\left(I^{\tau}-\hat{L}_{t_{k}, 0}^{\tau}\right)^{-1}\left(r^{\tau}+\frac{1}{k}\right)-\left(I^{\tau}-N^{\tau}\right)^{-1} r^{\tau}\right\|_{\tau} \\
& \leqslant\left\|\left(I^{\tau}-\hat{L}_{t_{k}, 0}^{\tau}\right)^{-1} r^{\tau}-\left(I^{\tau}-N^{\tau}\right)^{-1} r^{\tau}\right\|_{\tau}+\left\|\left(I^{\tau}-\hat{L}_{t_{k}, 0}^{\tau}\right)^{-1}\left(\frac{1}{k}\right)\right\|_{\tau} \\
& \leqslant\left\|\left(I^{\tau}-L_{t_{k}, 0}^{\tau}\right)^{-1} r^{\tau}-\left(I^{\tau}-N^{\tau}\right)^{-1} r^{\tau}\right\|_{\tau}+M \frac{1}{k},
\end{aligned}
$$

which tends to zero as $k \rightarrow \infty$. Hence (2.9) is true. Now we see that

$$
0 \geqslant-q(t)=\varepsilon(t)=\lim p_{k}(t) \geqslant 0, \quad t \in[0, \tau],
$$

namely $q(t)=0, t \in[0, \tau]$. Since $\tau$ is arbitrary the proof of the lemma is complete.

Proof of TheOrem 2.4. We shall first prove that $x$ is the unique solution of the equation $u=T u$. To do this, let $y$ be another solution and let $\tau>0$. We set $y_{1}(t)=y(t), t \leqslant \tau, y_{1}(t)=y(\tau)-x(\tau)+x(t), t>\tau$. Let $K$ be a positive number such that $|x(s)-y(s)| \leqslant K, s \in[0, \tau]$. Then we have $\left|x(s)-y_{1}(s)\right| \leqslant K$ for all $s \geqslant 0$. We set $q(t)=\left|x(t)-y_{1}(t)\right|, t \geqslant 0$. Then by (2.3) we get $q(t) \leqslant\left(L_{K} q\right)(t)$, $t \geqslant 0$, for an operator $L_{K}$ satisfying the conditions stated in the theorem. Since $q(0)=0$, by Lemma 2.6, we conclude that $q(t)=0, t \geqslant 0$. Since $\tau$ is arbitrary we have $x=y$, namely the equation $u=T u$ admits the unique solution $x$.

Let $(\bar{x}, S)$ be a point in the (sequential) closure of $\left\{\left(x_{t}, T_{t, x}\right): t \geqslant 0\right\}$. To prove the theorem it is enough to show that $S$ satisfies a condition similar to (2.3), for, by Lemma 2.6, and the preceding argument, it follows that $\bar{x}$ is the unique solution of $u=S u$. Let $y$ be in $C_{n}(\bar{x}(0)) \equiv C_{n}(v(S))$ with $|\bar{x}(s)-y(s)| \leqslant K, s \geqslant 0$, for a certain positive $K$. Let also $\left\{t_{n}\right\}$ be a sequence in $\mathbf{R}^{+}$such that $T_{t_{n}, x} \rightarrow S$ and $x_{t_{n}} \rightarrow \bar{x}$. Setting $y^{n}(t)=\left(1-e^{-n t}\right)[y(t)-\bar{x}(t)]+x\left(t_{n}+t\right), t \geqslant 0, n=1,2, \ldots$, we observe that $y^{n}(0)=x\left(t_{n}\right), y^{n} \rightarrow y$, as $n \rightarrow \infty$ and $\left|\mu_{t_{n}, x} x_{t_{n}}(s)-\mu_{t_{n}, x} y^{n}(s)\right| \leqslant K$, for all $n=1,2, \ldots$ and $s \geqslant 0$. Applying (2.3) we get

$$
\begin{aligned}
\mid\left(T_{t_{n}, x} x_{t_{n}}\right) & (t)-\left(T_{t_{n}, x} y^{n}\right)(t) \mid \\
& =\left|\left(T \mu_{t_{n}, x} x_{t_{n}}\right)\left(t_{n}+t\right)-\left(T \mu_{t_{n}, x} y^{n}\right)\left(t_{n}+t\right)\right| \\
& \leqslant L\left(\left|\mu_{t_{n}, x} x_{t_{n}}-\mu_{t_{n}, x} y^{n}\right|\right)\left(t_{n}+t\right) \\
& =L\left(\mu_{t_{n}, 0}\left|x_{t_{n}}-y^{n}\right|\right)\left(t_{n}+t\right) \\
& =L_{t_{n}, 0}\left(\left|x_{t_{n}}-y^{n}\right|\right)(t), \quad n=1,2, \ldots, t \geqslant 0,
\end{aligned}
$$

where $L$ is an operator which corresponds to $K$ and satisfies the inequality (2.3). By our assumption on the sequence $\left\{L_{t_{n}, 0}\right\}$ we can assume that there exists an operator $N$ such that $L_{t_{n}, 0} \rightarrow N$. Therefore by (2.10) we obtain

$$
|(S \bar{x})(t)-(S y)(t)| \leqslant N(|\bar{x}-y|)(t), \quad t \geqslant 0,
$$

which proves the theorem.

By using Lemma 2.6 it is also easy to prove the following theorem which would be useful in studying stability via the limiting equations (see, e.g. [1,3]). 
TheOREM 2.7. Let $T$ be an operator in T. Assume that for each $K>0$ there is an operator $L$ as in Theorem 2.4 such that (2.3) holds for all $x, y$ in $C_{n}(v(T))$, with $|x(t)|$, $|y(t)| \leqslant K, t \geqslant 0$. Assume also that $L$ satisfies (2.1) for a certain $M>0$ and that the set $\left\{L_{t, 0}: t \geqslant 0\right\}$ is precompact. Then for any operator $S$ such that $S=\lim T_{t_{k}, y_{k}}$, for $a$ certain sequence $\left\{t_{k}\right\} \subset \mathbf{R}^{+}$and $\left\{y_{k}\right\} \subset C_{n}(v(T))$, with $\left|y_{k}(t)\right| \leqslant \alpha, t \geqslant 0, k=$ $1,2, \ldots, \alpha>0$, the operator equation $u=S u$ admits at most one solution.

Proof. It is enough to show that $S$ satisfies an inequality similar to (2.3). This can be easily seen by using a procedure analogous to that used for the proof of (2.11). Then we apply Lemma 2.6 again.

REMARK 2.8. When we say that the set $\left\{L_{t, 0}: t \geqslant 0\right\}$ is precompact we mean that whenever $\left\{t_{m}\right\}$ is a sequence in $[0, \infty)$ the sequence $\left\{L_{t_{m}, 0}\right\}$ has a subsequence which converges to a continuous causal operator on $C_{1}(0)$ to $C_{1}(0)$.

\section{REFERENCES}

1. Z. Artstein, Uniform asymptotic stability via the limiting equations, J. Differential Equations 27 (1978), $172-189$.

2. N. Dunford and J. T. Schwartz, Linear operators, Part I, Interscience, New York, 1958.

3. G. Karakostas, Asymptotic behavior of causal operator equations, Ph. D. dissertation, The Weizmann Institute of Science, Rehovot, Israel, 1979.

4. ___ Causal operators and topological dynamics, Ann. Mat. Pure Appl. (to appear).

5. Limiting and full limiting equations of causal operator equations (to appear).

6. R. K. Miller and G. R. Sell, Volterra integral equations and topological dynamics, Mem. Amer. Math. Soc. No. 102, 1970.

7. L. W. Neustadt, On the solutions of certain integral-like operator equations. Existence, uniqueness and dependence theorems, Arch. Rational Mech. Anal. 38 (1970), 131-160.

8. G. R. Sell, Nonautonomous differential equations and topological dynamics. I. The basic theory, Trans. Amer. Math. Soc. 127 (1967), 241-262.

Department of MAThematics, University of IOANNina, IoANnina, Greece 